\title{
Trinucleotide repeat
}

National Cancer Institute ( $\mathrm{NCl})$

\section{Source}

National Cancer Institute (NCI). Trinucleotide Repeat.

Sequences of 3 nucleotides repeated in tandem on the same contiguous section of chromosome. A certain amount of normal (polymorphic) variation in repeat number with no clinical significance commonly occurs between individuals; however, repeat numbers over a certain threshold can, in some cases, lead to adverse effects on the function of the gene, resulting in genetic disease. 